\title{
Convergence of Cauchy-Riemann Sums to Cauchy-Riemann Integrals ${ }^{1}$
}

\section{Otto Szász and John Todd}

Two general theorems giving conditions to insure the truth of the relation

$$
\lim _{h \rightarrow 0} \sum_{n=1}^{\infty} f(n h)=\int_{0}^{\infty} f(x) d x
$$

are established. In addition, several cases involving Bessel functions are discussed.

\section{Introduction}

We are concerned with the validity of the equation

$$
\lim _{h \rightarrow+0} h \sum_{v=1}^{\infty} f(v h)=\int_{0}^{\infty} f(x) d x
$$

where the integral is taken in the Cauchy-Riemann sense, that is,

$$
\int_{0}^{\infty} f(x) d x=\lim _{\substack{\epsilon \rightarrow 0 \\ \omega \rightarrow \infty}} \int_{\epsilon}^{\omega} f(x) d x .
$$

There are cases when (1) is meaningless if the sum and the integral are interpreted in the ordinary way, but it becomes meaningful and correct if suitable summability methods are employed. A very simple case is when

$$
f(x)=\sin x,
$$

in which (1) is true if both the series and the integral are interpreted in the $(\mathbf{C}, 1)$ sense. We do not propose to consider such cases at present. We shall also concern ourselves mainly with the case when the integrand has no singularity at the origin. Detailed investigations of the case when there is a singularity at the origin have been carried out by $\mathrm{A}$. Wintner $[8]^{2}$ and $A$. E. Ingham [3].

The problem was studied by Bromwich and Hardy [1] in 1908, and some of the results they obtained are now found in textbooks. Their results are of the following type:

If $f(x)$ decreases steadily and if the integral exists, then the series converges and (1) holds.

If $\varphi(x)$ is continuous and decreases steadily to zero then (1) is true for $f(x)=\varphi(x) \sin x$.

If $\varphi(x)$ is continuous and decreases steadily to zero and if $\int_{0}^{\infty} \varphi(x) d x$ exists, if furthermore $F(x)$ is a bounded continuous function, then (1) is true for $f(x)=\varphi(x) F(x)$.

1 The preparation of this paper was sponsored (in part) by the Office of Naval Research.

${ }^{2}$ Figures in brackets indicate the literature references at the end of this paper.
We note that (2) covers such cases as $f(x)=x e^{-x}$, where $f^{\prime}(x)<0$ for $x>1$; that (3) covers such cases as $\varphi(x)=x^{-\alpha}, 0<\alpha \leq 1$ (which is continuous except at $x=0)$ and that $(4)$ covers such cases as $\varphi(x)=x^{-2}$, $F(x)=\sin ^{2} x$.

The result (2) has been generalized to the quasimonotone case by O. Szász [6] as follows:

If for some $\alpha \geq 0$, for $x \geq 1$ and $0<y<1$, we have

$$
0<f(x+y) \leq\left(1+\frac{\alpha}{x}\right) f(x),
$$

and if the integral exists, then (1) holds.

Krishnan [9] has pointed out that in certain (nontrivial) cases a stronger result than (1) is true, namely,

$$
h \sum f(\nu h)=\int_{0}^{\infty} f(x) d x
$$

for all sufficiently small positive $h$; indeed:

If $f(x)$ is a function whose Fourier transform vanishes outside a finite interval $[-a, a]$ and if the Poisson formula can be applied, then we have (for all $\theta$ )

$$
h \sum_{\nu=-\infty}^{\infty} f(\nu \alpha+\theta)=h \sum_{\nu=-\infty}^{\infty} f(\nu h)=\int_{-\infty}^{+\infty} f(x) d x,
$$

provided $0<h<2 \pi / a$.

A simple case when this applies is when $f(x)=x^{-2} \sin ^{2} x$. We use this technique to give an alternative proof of one of our results on Bessel functions.

In sections 2 and 3 we give two sets of conditions that are sufficient to insure the truth of (1) and that include many of the known cases. The remainder of the paper, which is independent of these two sections, gives discussions, by various methods, of special cases, mainly involving Bessel functions, in which (1) is true.

\section{2. $x f(x)=\psi(x)$, a Periodic Function}

Our first theorem asserts the truth of (1), where $f(x)=x^{-1} \psi(x)$ under certain conditions on $\psi(x)$. The simplest case is that when $\psi(x)=\sin x$. 
Theorem 1. Let

$$
\psi(x)=\sum_{n=1}^{\infty} b_{n} \sin n x, \quad \sum\left|b_{n}\right|<\infty .
$$

Let the Cauchy-Riemann integral

$$
\int_{\rightarrow 0}^{\pi} \frac{\psi(t)}{t} d t
$$

exist. Then (1) holds for $f(x)=x^{-1} \psi(x)$, i. e., we have

$$
\lim _{h \rightarrow+0} h \sum_{n=1}^{\infty} \frac{\psi(n h)}{n h}=\int_{\rightarrow 0}^{\infty} \frac{\psi(x)}{x} d x .
$$

Proof. We begin by establishing convergence of the series on the left of (10). We do this by first establishing that it is summable- $A$ and then noticing that since $\psi$ is bounded, its general term is $O\left(n^{-1}\right)$, and we can therefore use a standard Tauberian theorem [10, p. 501]. We therefore consider

$$
\left.\begin{array}{rl}
\sum_{n=1}^{\infty} \frac{\psi(n h)}{n} r^{n} & =\sum_{n=1}^{\infty} \frac{r^{n}}{n} \sum_{\nu=1}^{\infty} b_{\nu} \sin \nu n h \\
& =\sum_{\nu=1}^{\infty} b_{\nu} \sum_{n=1}^{\infty} \frac{r^{n}}{n} \sin \nu n h .
\end{array}\right\}
$$

This interchange of summation being permissible because of the absolute convergence of the double series

$$
\sum \sum\left|b_{\nu}\right|\left(r^{n} / n\right)=\sum\left|b_{\nu}\right| \sum\left(r^{n} / n\right)
$$

whenever $0 \leq r<1$. We now require some elementary properties of the inner series in (11). It is well known [11, p. 285] that

$$
\sum_{n=1}^{\infty} \frac{r^{n}}{n} \sin n x=\arctan \frac{r \sin x}{1-r \cos x}
$$

when $0 \leq r<1$. This gives

$$
\sum_{n=1}^{\infty} \frac{r^{n}}{n} \sin \nu n h=\arctan \frac{r \sin \nu h}{1-r \cos \nu h}
$$

when $0 \leq r<1$. Since for $r=0$, the left-hand side is zero, we must choose that determination of the arctan that vanishes for $r=0$. Since the denominator $1-r \cos \nu h$ never vanishes $(0 \leq r<1, h \rightarrow+0, \nu=1$, $2, \ldots$ ) we cannot leave this branch and, in particular, we have

$$
\left|\sum_{n=1}^{\infty} \frac{r^{n}}{n} \sin \nu n h\right|=\left|\arctan \frac{r \sin \nu h}{1-r \cos \nu h}\right| \leq \frac{1}{2} \pi .
$$

Hence, we have

$$
\sum_{n=1}^{\infty} \frac{\psi(n h)}{n} r^{n}=\sum_{\nu=1}^{\infty} b_{\nu} \arctan \frac{r \sin \nu h}{1-r \cos \nu h}
$$

where the series on the right is, for fixed $h>0$, uniformly and absolutely convergent in $0 \leq r<1$, being majorized by $\frac{1}{2} \pi \sum_{\nu=1}^{\infty}\left|b_{\nu}\right|$.

Hence, as $r \rightarrow 1$, we have [10, p. 339]

$$
\begin{aligned}
\sum_{\nu=1}^{\infty} b_{\nu} \arctan \frac{r \sin \nu h}{1-r \cos \nu h} & \rightarrow \sum_{\nu=1}^{\infty} b_{\nu} \arctan \frac{\sin \nu h}{1-\cos \nu h} \\
& =\sum_{\nu=1}^{\infty} b_{\nu} \arctan \left(\cot \frac{1}{2} \nu h\right) .
\end{aligned}
$$

This means that $\sum_{n=1}^{\infty} \psi(n h) / n$ is, for all $h>0$, summable- $A$, and therefore convergent, to the sum

$$
\sum_{\nu=1}^{\infty} b_{\nu} \arctan \left(\cot \frac{1}{2} \nu h\right)
$$

We shall now show that, as $h \rightarrow 0$,

$$
\sum_{\nu=1}^{\infty} b_{\nu} \arctan \left(\cot \frac{1}{2} \nu h\right) \rightarrow \frac{1}{2} \pi \sum_{\nu=1}^{\infty} b_{\nu}
$$

The difference between these two expressions can be written as

$$
\begin{aligned}
& \sum_{\nu=1}^{N}\left\{b_{\nu} \arctan \left(\cot \frac{1}{2} \nu h\right)-\frac{1}{2} \pi b_{\nu}\right\}+ \\
& \sum_{\nu=N+1}^{\infty} b_{\nu} \arctan \left(\cot \frac{1}{2} \nu h\right)-\frac{1}{2} \pi \sum_{N+1}^{\infty} b_{\nu} .
\end{aligned}
$$

We first choose $N$ so large that $\frac{1}{2} \pi \sum_{N+1}^{\infty} b_{\nu}$ (and therefore also $\sum_{\nu=N+1}^{\infty} b_{\nu} \arctan \left(\cot \frac{1}{2} \nu h\right)$ is small. With $N$ fixed we then can choose $h$ so small that the first sum in (13) is small. This establishes (12). To complete the proof of Theorem 1 we have first to establish the existence of the integral on the right of (10) and then show that its value is $\frac{1}{2} \pi \sum_{\nu=1}^{\infty} b_{\nu}$. Our hypothesis (9) insures convergence at the lower limit. Integrating by parts, we see that

$$
\int_{\pi}^{u} \frac{\psi(x)}{x} d x=\frac{\psi_{1}(u)}{u}+\int_{\pi}^{u} \frac{\psi_{1}(x)}{x^{2}} d x,
$$

where

$$
\psi_{1}(u)=\int_{\pi}^{u} \psi(x) d x
$$

Since $\psi_{1}(x)=O(1)$ by periodicity, and since $\int^{\infty} x^{-2} d x$ is convergent, we can let $u \rightarrow \infty$ in (14) to obtain

$$
\int_{\pi}^{\infty} \frac{\psi(x)}{x^{2}} d x=\int_{\pi}^{\infty} \frac{\psi_{1}(x)}{x^{2}} d x .
$$


Thus the existence of

is established.

We now consider

$$
\left.\begin{array}{rl}
\int_{t}^{\infty} \frac{\psi(x)}{x} d x & =\int_{t}^{\infty} \frac{\sum_{\nu=1}^{\infty} b_{\nu} \sin \nu x}{x} \mathrm{~d} x \\
& =\sum_{\nu=1}^{\infty} b_{\nu} \int_{t}^{\infty} \frac{\sin \nu x}{x} d x
\end{array}\right\}
$$

This inversion may be justified as follows. We use the fact that

$S i(x)=\int_{x}^{\infty} \frac{\sin t}{t} d t=\left[\frac{-\cos t}{t}\right]_{x}^{\infty}-\int_{x}^{\infty} \frac{\cos t d t}{t^{2}}=O\left(x^{-1}\right)$

as $x \rightarrow \infty$. What we have to show is that

$$
I_{1}=\int_{t}^{T} \sum_{\nu=N}^{\infty} \frac{b_{\nu} \sin \nu x}{x} d x=\int_{t}^{T} \frac{1}{x} \sum_{\nu=N}^{\infty} b_{\nu} \sin \nu x d x
$$

and

$$
I_{2}=\sum_{\nu=1}^{N} b_{\nu} \int_{T}^{\infty} \frac{\sin \nu x}{x} d x
$$

are both small for $N, T$ sufficiently large. Since $\sum b_{\nu}$ is absolutely convergent, and since for all $\nu$, $\int_{T}^{\infty} \frac{\sin \nu x}{x} d x=O\left(T^{-1}\right)$, it follows that $I_{2}=O\left(T^{-1}\right)$, i. e., small if $T$ is large uniformly for all $N$. Fixing $T$ we can choose $N$ so large that $I_{1}$ is small. This establishes (15).

We can let $t \rightarrow 0$ in (15) since the series on the right is uniformly convergent in $t$. This gives

$$
\int_{\rightarrow 0}^{\infty} \frac{\psi(x)}{x} d x=\sum_{\nu=1}^{\infty} b_{\nu} \int_{0}^{\infty} \frac{\sin \nu x}{x} d x=\frac{1}{2} \pi \sum_{\nu=1}^{\infty} b_{\nu}
$$

and thereby completes the proof of Theorem 1 .

Remark: An example that is not covered by this theorem is the case when $b_{n}=(-1)^{n} / n$. Here

$$
\psi(t)=\sum b_{n} \sin n t=\pi\left\{\left[t+\frac{1}{2}\right]-t\right\}
$$

if $t+\frac{1}{2}$ is not an integer and $\psi(t)=0$ if $t+\frac{1}{2}$ is an integer, and although it is clear that $\int_{0}^{\infty} f(x) d x$ exists, the question of the truth or falsity of (1) seems to be rather deep.

\section{3. $x f(x)=\psi(x)$, a Fourier Integral}

Our second theorem asserts the truth of (1) when $x f(x)$ is the sine transform of an absolutely integrable function $\eta(t)$. This, when we take $\eta(t)=0, \quad t<0, \quad \eta(t)=\frac{1}{2}, \quad 0 \leq t \leq 2, \quad \eta(t)=0, \quad t>2$

reduces to the case

$$
f(x)=\sin ^{2} x / x^{2},
$$

which has been mentioned by Hardy and Bromwich [1] and by Krishnan [9].

Theorem 2. Let

where

$$
f(x)=\frac{1}{x} \int_{0}^{\infty} \eta(t) \sin x t d x,
$$

$$
\int_{0}^{\infty}|\eta(t)| d t
$$

is finite. Then (1) is true.

Proof. We have

$$
\begin{aligned}
h \sum_{\nu=1}^{\infty} f(\nu h) & =\sum_{\nu=1}^{\infty} \frac{1}{\nu} \int_{0}^{\infty} \sin \nu h t \eta(t) d t \\
& =\int_{0}^{\infty} \sum_{\nu=1}^{\infty} \frac{1}{\nu} \sin \nu h t \eta(t) d t .
\end{aligned}
$$

This interchange of summation and integration is legitimate because $\sum \nu^{-1} \sin \nu h t$ is convergent and its partial sums are uniformly bounded.

It is well known [10, p. 523] that for $x$ positive and not an integer,

$$
\sum_{\nu=1}^{\infty} \nu^{-1} \sin 2 \pi \nu x=-\pi\left\{x-[x]-\frac{\mathrm{\tau}}{2}\right\},
$$

where $[x]$ denotes the integral part of $x$. This gives

$$
\sum \nu^{-1} \sin \nu h t=-\pi\left\{\frac{h t}{2 \pi}-\left[\frac{h t}{2 \pi}\right]-\frac{1}{2}\right\} .
$$

Substituting, we find

$$
\begin{aligned}
& \int_{0}^{\infty}\left\{\sum_{\nu=1}^{\infty} \nu^{-1} \sin \nu h t\right\} \eta(t) d t \\
= & -\pi \int_{0}^{\infty}\left\{\frac{h t}{2 \pi}-\left[\frac{h t}{2 \pi}\right]-\frac{1}{2}\right\} \eta(t) d t \\
= & \frac{1}{2} \pi \int_{0}^{\infty} \eta(t) d t-\pi \int_{0}^{\infty}\left\{\frac{h t}{2 \pi}-\left[\frac{h t}{2 \pi}\right]\right\} \eta(t) d t .
\end{aligned}
$$

We shall show that the second term on the right tends to zero with $h$. To do this, write

$$
\begin{aligned}
& \int_{0}^{\infty}\left\{\frac{h t}{2 \pi}-\left[\frac{h t}{2 \pi}\right]\right\} \eta(t) d t \\
= & \int_{0}^{\omega}\left\{\frac{h t}{2 \pi}-\left[\frac{h t}{2 \pi}\right]\right\} \eta(t) d t+\int_{\omega}^{\infty}\left\{\frac{h t}{2 \pi}-\left[\frac{h t}{2 \pi}\right]\right\} \eta(t) d t \\
= & I_{1}(h)+I_{2}(h),
\end{aligned}
$$

say. We can choose and fix $\omega$ so large that 


$$
\left|I_{2}(h)\right| \leq \int_{\omega}^{\infty}|\eta(t)| d t
$$

is small for all $h$. Then we can choose $h$ so small that

$$
\frac{h t}{2 \pi}-\left[\frac{h t}{2 \pi}\right] \leq \frac{h t}{2 \pi}
$$

is itself small enough to insure that $\left|I_{1}(h)\right|$ is small.

Hence

$$
h \sum_{\nu=1}^{\infty} f(\nu h) \rightarrow \frac{1}{2} \pi \int_{0}^{\infty} \eta(t) d t .
$$

To complete the proof we have to show that

$$
\int_{0}^{\infty} f(x) d x=\frac{1}{2} \pi \int_{0}^{\infty} \eta(t) d t
$$

but this follows easily, since

$$
\begin{aligned}
\int_{0}^{\infty} f(x) d x & =\int_{0}^{\infty} \int_{0}^{\infty} \frac{\sin x t}{x} \eta(t) d t d x \\
& =\int_{0}^{\infty}\left[\int_{0}^{\infty} \frac{\sin x t}{x} d x\right] \eta(t) d t \\
& =\frac{1}{2} \pi \int_{0}^{\infty} \eta(t) d t
\end{aligned}
$$

the change of order of integration being permissible, since $\int_{0}^{\infty} \eta(t) d t$ is, by hypothesis, absolutely convergent.

\section{The Cases $f(x)=J_{0}(x)$ and $f(x)=K_{0}(x)$}

It is known [5, p. 336] that

$$
\sum_{n=0}^{\infty} J_{0}(n h)=\frac{1}{2}+h^{-1}
$$

and so, as $h \rightarrow 0$,

$$
h \sum_{n=0}^{\infty} J_{0}(n h) \rightarrow 1
$$

Since also [2, p. 96]

$$
\int_{0}^{\infty} J_{0}(x) d x=1
$$

the relation (1) is true in this case.

The result (16) can be established directly in the following manner. From the asymptotic formula

$$
J_{0}(x)=\left(\frac{2}{\pi x}\right)^{\frac{1}{2}} \sin \left(x+\frac{1}{4} \pi\right)+O\left(x^{-3 / 2}\right),
$$

using partial summation, it can be shown that the series $\sum_{n=0}^{\infty} J_{0}(n h)$ converges for $0<h<\pi$ (and, indeed, converges uniformly in every closed subinterval). We evaluate the sum of this series by determining its $(C, 1)$ sum, using the integral representation

$$
J_{0}(x)=\frac{2}{\pi} \int_{0}^{\pi / 2} \cos (x \cos t) d t
$$

and the facts that

$$
\begin{gathered}
C_{n}(x) \equiv \sum_{\nu=0}^{n} \cos \nu x=\frac{1}{2}+\frac{\sin \left(n+\frac{1}{2}\right) x}{2 \sin \frac{1}{2} x}, \\
\frac{1}{n+1} \sum_{\nu=0}^{n} C_{\nu}(x)=\frac{1}{2}+\frac{\sin ^{2}\left(n+\frac{1}{2}\right) x}{2(n+1) \sin ^{2} \frac{1}{2} x} .
\end{gathered}
$$

From (17), (18), and (19) we obtain

$$
\begin{aligned}
s_{n}(h) & =\sum_{\nu=0}^{n} J_{0}(\nu h)=\frac{2}{\pi} \int_{0}^{\pi / 2}\left\{\frac{1}{2}+\frac{\sin \frac{1}{2}(n+1) h \cos t}{2 \sin \left(\frac{1}{2} h \cos t\right)}\right\} d t \\
\sigma_{n}(h) & \equiv \frac{1}{n+1} \sum_{\nu=0}^{n} s_{\nu}(h) \\
& =\frac{1}{\pi} \int_{0}^{\pi / 2}\left\{1+\frac{\sin ^{2}\left[\frac{1}{2}(n+1) h \cos t\right]}{(n+1) \sin ^{2}\left(\frac{1}{2} h \cos t\right)}\right\} d t
\end{aligned}
$$

Now $\sin ^{-2} x=\left(1 / x^{2}\right)+\mathrm{O}(1)$ as $x \rightarrow 0$, and so, for fixed $h>0$ we find

$$
\begin{aligned}
\sigma_{n}(h)= & \frac{1}{2}+ \\
& \frac{4}{\pi(n+1) h^{2}} \int_{0}^{\pi / 2} \frac{\sin ^{2}\left[\frac{1}{2}(n+1) h \sin t\right]}{\sin ^{2} t} d t+O\left(\frac{1}{n}\right) \\
= & \frac{1}{2}+R_{1}(n)+R_{2}(n)+O\left(n^{-1}\right),
\end{aligned}
$$

where $R_{1}$ and $R_{2}$ are the contributions from the ranges $(0, \pi / 4)$ and $(\pi / 4, \pi / 2)$, respectively. Clearly,

$$
R_{2}(n)<\frac{4}{\pi n h^{2} \sin ^{2} \frac{\pi}{4}}=O\left(n^{-1}\right) .
$$

To deal with $R_{1}$ we change the variable $t$ to $u=$ $\frac{1}{2}(n+1) h \sin t$; we find, writing $h(n+1) 2^{-3 / 2}=k$, that 


$$
\begin{aligned}
R_{1}(n)= & \frac{2}{\pi h} \int_{0}^{k} \frac{\sin ^{2} u d u}{u^{2}\left\{1-\left(\frac{2 u}{(n+1) h}\right)^{2}\right\}^{\frac{1}{2}}} \\
= & \frac{2}{\pi h} \int_{0}^{k}\left(\frac{\sin u}{u}\right)^{2} d u+ \\
& \frac{2}{\pi h} \int_{0}^{k}\left(\frac{\sin u}{u}\right)^{2}\left\{\left[1-\left(\frac{2 u}{(n+1) h}\right)^{2}\right]^{-\frac{1}{2}}-1\right\} d u .
\end{aligned}
$$

As $n \rightarrow \infty, k \rightarrow \infty$ and the first term tends to $h^{-1}$ and is indeed $h^{-1}+O\left(n^{-2}\right)$.

Let $v=[2 u /(n+1) h]^{2}$; then in the range $0 \leq u \leq k$ we have $0 \leq v \leq \frac{1}{2}$, and

$$
\begin{aligned}
(1-v)^{-\frac{1}{2}}-1 & =\frac{(1-v)^{-1}-1}{1+(1-v)^{-\frac{1}{2}}} \\
& =\frac{v}{(1-v)\left\{1+(1-v)^{-\frac{1}{2}}\right\}} \\
& <2 v=\frac{8 u^{2}}{(n+1)^{2} h^{2}} .
\end{aligned}
$$

This means that the second term is $O\left(n^{-2}\right)$ as $n \rightarrow \infty$.

Gathering our results together, we have

$$
\sigma_{n}(h)=\frac{1}{2}+h^{-1}+O\left(n^{-1}\right) .
$$

This means that the series $\sum J_{0}(n h)$ is summable $(C, 1)$ to the sum $\frac{1}{2}+h^{-1}$, when $h>0$. This completes the sketch of the proof of (16).

To deal with the case $f(x)=K_{0}(x)$ we use the formula [13, p. 301]

$$
\begin{aligned}
\sum_{n=1}^{\infty} K_{0}(n h)= & \frac{\pi}{2 h}+\pi \sum_{n=1}^{\infty}\left\{\frac{1}{\sqrt{h^{2}+4 n^{2} h^{2}}}-\frac{1}{2 n \pi}\right\}+\frac{1}{2} \gamma+ \\
& \frac{1}{2} \ln h-\frac{1}{2} \log 4 \pi,
\end{aligned}
$$

which leads to

$$
\lim _{h \rightarrow 0} h \sum_{n=1}^{\infty} K_{0}(n h)=\frac{1}{2} \pi .
$$

Since [12, p. 388] we have

$$
\int_{0}^{\infty} K_{0}(x) d x=\frac{1}{2} \pi
$$

(1) is true in this case. We note that here we have a (logarithmic) singularity at the origin, and have used a summation starting at 1 and not at 0 to avoid trouble.

\section{The Cases $f(x)=J_{0}(x) \cos x t$ and $f(x)=$ $J_{0}(x) \sin x t$}

It is known [4, p. 59] that for $h>0,0 \leq t<1$, we have

$$
\sum_{n=1}^{\infty} J_{0}(n h) \cos (n h t)=-\frac{1}{2}+\frac{1}{h \sqrt{1-t^{2}}} .
$$

Multiplying by $h$ and then letting $h \rightarrow 0$ we find, for $0 \leq t<1$,

$$
h \sum_{n=1}^{\infty} J_{0}(n h) \cos (n h t) \rightarrow \frac{1}{\sqrt{1-t^{2}}} .
$$

On the other hand [4, p. 51], we have, for $0 \leq t<1$,

$$
\int_{0}^{\infty} J_{0}(x) \cos x t d x=\frac{1}{\sqrt{1-t^{2}}}
$$

and so (1) is true.

The case when we consider

$$
f(x)=J_{0}(x) \sin x t
$$

is slightly more complicated. It is known [4, p. 59] that for $h>0,0 \leq t<1$, we have

$$
\begin{aligned}
& \sum_{n=1}^{\infty} J_{0}(n h) \sin (n h t) \\
= & \sum_{n=1}^{\infty}\left\{\left[(2 \pi n+t h)^{2}-h^{2}\right]^{-1 / 2}-\left[(2 \pi n-t h)^{2}-h^{2}\right]^{-1 / 2}\right\} \\
= & -\sum_{n=1}^{\infty}\{8 \pi n t h / D\},
\end{aligned}
$$

where

$$
\begin{aligned}
D= & {\left[(2 \pi n+t h)^{2}-h^{2}\right]^{1 / 2}\left[(2 \pi n-t h)^{2}-h^{2}\right]^{1 / 2} \times } \\
& \left\{\left[(2 \pi n+t h)^{2}-h^{2}\right]^{1 / 2}+\left[(2 \pi n-t h)^{2}-h^{2}\right]^{1 / 2}\right\} .
\end{aligned}
$$

Now for small $h$ and fixed $t$

$$
\frac{n t h}{D}=O\left(\frac{h}{n^{2}}\right)
$$

Hence, as $h \rightarrow 0$,

$$
h \sum_{n=1}^{\infty} J_{0}(n h) \sin (n h t) \rightarrow 0
$$

On the other hand, it is known [4, p. 51] that for $0 \leq t<1$

$$
\int_{0}^{\infty} J_{0}(x) \sin x t d x=0
$$

Thus (1) holds in this case also.

$$
\text { 6. The Case } f(x)=\underset{\mu}{\Lambda_{\mu}(x)=(1 / 2}\left(2^{\mu} x^{-\mu}\right) \Gamma(\mu+1) J_{\mu}(x),
$$

It is known [2, p. 91] that

$$
\sum_{n=0}^{\infty} \Lambda_{\mu}(n h)=\frac{\sqrt{\pi}}{h} \frac{\Gamma(\mu+1)}{\Gamma\left(\mu+\frac{1}{2}\right)}
$$


and so, as $h \rightarrow 0$,

$$
h \sum_{n=0}^{\infty} \Lambda_{\mu}(n h) \rightarrow \frac{\sqrt{\pi} \Gamma(\mu+1)}{\Gamma\left(\mu+\frac{1}{2}\right)} .
$$

The convergence of this series can be established in a similar manner to that used in the case $\mu=0$.

Since also $[12$, p. 391] or $[7$, p. 182]

$$
\int_{0}^{\infty} \Lambda_{\mu}(x) d x=\frac{\sqrt{\pi} \Gamma(\mu+1)}{\Gamma\left(\mu+\frac{1}{2}\right)},
$$

the relation (1) is true in this case, and is a generalization of that discussed in section 4. This case, when $\mu=1 / 2$, reduces to the case $f(x)=\sin x / x$.

We shall now discuss this case using the Poisson summation formula

$$
\sqrt{\beta}\left\{\frac{1}{2} F_{c}(0)+\sum_{n=1}^{\infty} F_{c}(n \beta)\right\}=\sqrt{\alpha}\left\{\frac{1}{2} f(0)+\sum_{n=1}^{\infty} f(n \alpha)\right\},
$$

where $\alpha \beta=2 \pi, \alpha>0$ and $F_{c}$ is the cosine transform of $f$ defined by

$$
F_{c}(x)=\sqrt{\frac{2}{\pi}} \int_{0}^{\rightarrow \infty} f(t) \cos x t d t .
$$

This formula is true, for instance [7, p. 60 to 64], provided that $f(x)$ is continuous and of bounded variation in $0 \leq x<\infty$, that $f(x) \rightarrow 0$ as $x \rightarrow \infty$ and that the Cauchy-Riemann integral $\int_{0}^{\rightarrow \infty} f(t) d t$ exists.

These conditions are satisfied when $\mu>\frac{1}{2}$ if

$$
\begin{array}{ll}
f(x)=\frac{2^{\frac{1}{2}-\mu}}{\Gamma\left(\mu+\frac{1}{2}\right)}\left(1-x^{2}\right)^{\mu-\frac{1}{2}}, & 0<x<1 \\
f(x)=0, & x \geq 1
\end{array}
$$

so that $[7$, p. 65$]$

$$
F_{c}(x)=\frac{J_{\mu}(x)}{x^{\mu}}=\frac{2^{-\mu}}{\Gamma(\mu+1)} \Lambda_{\mu}(x) .
$$

In this case (21) gives

$$
\frac{1}{2^{\mu} \Gamma(\mu+1)}\left\{\frac{1}{2}+\sum_{n=1}^{\infty} \Lambda_{\mu}(n \beta)\right\}=
$$

$$
\left(\frac{\alpha}{\beta}\right)^{\frac{1}{2}} \frac{2^{\frac{1}{2}-\mu}}{\Gamma\left(\mu+\frac{1}{2}\right)}\left\{\frac{1}{2}+\sum_{\alpha n \leq 1}\left(1-n^{2} \alpha^{2}\right)^{\mu-\frac{1}{2}}\right\} .
$$

This formula is true as it stands for $\mu>\frac{1}{2}$; if $\mu=\frac{1}{2}$ and a term on the right corresponding to $n=\alpha^{-1}$ occurs, it must be halved; if $-\frac{1}{2}<\mu<\frac{1}{2}$, the formula remains true, provided that $\alpha$ is not the reciprocal of an integer.

We now assume $\alpha>1$, so that the sum on the right is empty; this means that

$$
\beta=2 \pi \alpha^{-1}<2 \pi
$$

In these circumstances (22) gives

$$
\frac{1}{2^{\mu} \Gamma(\mu+1)}\left\{\frac{1}{2}+\sum_{n=1}^{\infty} \Lambda_{\mu}(n \beta)\right\}=\left(\frac{2 \pi}{\beta^{2}}\right)^{\frac{1}{2}} \frac{1}{2^{\mu+\frac{1}{2}} \Gamma\left(\mu+\frac{1}{2}\right)},
$$

that is,

$$
\sum_{n=1}^{\infty} \Lambda_{\mu}(n \beta)=\frac{\sqrt{\pi}}{\beta} \frac{\Gamma(\mu+1)}{\Gamma\left(\mu+\frac{1}{2}\right)}-\frac{1}{2},
$$

which is (20).

\section{References}

[1] T. J. I'A. Bromwich and G. H. Hardy, The definition of an infinite integral as the limit of a finite or infinite series, Quart. J. Math. 39, 222 to 240 (1908).

[2] G. Doetsch, Summatorische Eigenschaften der Besselschen Funktionen, Compositio Mathematica 1, 85 to 97 (1935).

[3] A. E. Ingham, Improper integrals as limits of sums, J. London Math. Soc. 24, 44 to 50 (1949).

[4] W. Magnus and F. Oberhettinger, Formeln und Sätze für die speziellen Funkionen der mathematischen Physik 2d ed. (1948).

[5] N. Nielsen, Handbuch der Theorie der Cylinderfunktionen (Leipzig, 1904).

[6] O. Szász, Quasi-monotone series, Am. J. Math. 70, 203 to 206 (1948).

[7] E. C. Titchmarsh, Introduction to the theory of Fourier integrals (Oxford, 1948).

[8] Aurel Wintner, The sum formula of Euler-Maclaurin and the inversions of Fourier and Möbius, Am. J. Math. 69, 685 to 708 (1947).

[9] K. S. Krishnan, On the equivalence of certain infinite series and corresponding integrals, J. Indian Math. Soc. (New Series) 12, 79 to 88 (1948).

[10] K. Knopp, Theory and application of infinite series (London and Glasgow, 1928).

[11] T. J. I'A. Bromwich, Theory of infinite series (London, 1926).

[12] G. N. Watson, Bessel functions (Cambridge, 1948).

[13] G. N. Watson, Oxford Quart. J. 2, 298 to 309 (1931).

Los Angeles, July 20, 1950. 